OPEN ACCESS

Edited by:

Daniela Tropea,

Trinity College Dublin, Ireland

Reviewed by:

Dennis Qing Wang,

Third Affiliated Hospital of Sun

Yat-Sen University, China

Tatsuro Mutoh,

Fujita Health University, Japan

${ }^{*}$ Correspondence:

Yong Zhao

blys01@163.com

tThese authors have contributed equally to this work.

Received: 26 July 2016 Accepted: 29 September 2016 Published: 19 October 2016

Citation:

Wang S, Zhou Y, Yang B, Li L, YU S, Chen Y, Zhu J and Zhao Y (2016) C1q/Tumor Necrosis Factor-Related Protein-3 Attenuates Brain Injury after Intracerebral Hemorrhage via AMPK-Dependent Pathway in Rat. Front. Cell. Neurosci. 10:237. doi: 10.3389/fncel.2016.00237

\section{C1q/Tumor Necrosis Factor-Related Protein-3 Attenuates Brain Injury after Intracerebral Hemorrhage via AMPK-Dependent Pathway in Rat}

\author{
Shaohua Wang ${ }^{1,2 \dagger}$, Yang Zhou ${ }^{1,2 \dagger}$, Yang Bo ${ }^{1,2}$, Lingyu $L^{1,2}$, Shanshan $Y u^{1,2}$, \\ Yanlin Chen ${ }^{1,2}$, Jin Zhu ${ }^{1,2}$ and Yong Zhao ${ }^{1,2 *}$ \\ ${ }^{1}$ Department of Pathology, Chongqing Medical University, Chongqing, China, ${ }^{2}$ Key Laboratory of Neurobiology, Chongqing \\ Medical University, Chongqing, China
}

C1q/tumor necrosis factor (TNF)-related protein-3 (CTRP3) is a recently discovered adiponectin paralog with established metabolic regulatory properties. However, the role of CTRP3 in intracerebral hemorrhage $(\mathrm{ICH})$ is still mostly unresolved. The aim of the present report was to explore the possible neuroprotective effect of CTRP3 in an $\mathrm{ICH}$ rat model and to elucidate the fundamental mechanisms. $\mathrm{ICH}$ was induced in rats by intracerebral infusion of autologous arterial blood. The effects of exogenous CTRP3 (recombinant or lentivirus CTRP3) on brain injury were explored on day 7. Treatment with CTRP3 reduced brain edema, protected against disruption of the blood-brain barrier (BBB), improved neurological functions and promoted angiogenesis. Furthermore, CTRP3 greatly intensified phosphorylation of AMP-activated protein kinase (AMPK) in addition to expression of hypoxia inducing factor- $1 \alpha(\mathrm{HIF}-1 \alpha)$ and vascular endothelial growth factor (VEGF). Finally, the protective effects of CTRP3 could be blocked by either AMPK or VEGF inhibitors. Our findings give the first evidence that CTRP3 is a new proangiogenic and neuroprotective adipokine, which may exert its protective effects at least partly through an AMPK/HIF-1 $1 \alpha$ VEGF-dependent pathway, and suggest that CTRP3 may provide a new therapeutic strategy for $\mathrm{ICH}$.

Keywords: CTRP3, intracerebral hemorrhage, brain edema, blood-brain barrier disruption, neuroprotection, angiogenesis

\section{INTRODUCTION}

Intracerebral hemorrhage $(\mathrm{ICH})$ is responsible for about $10-15 \%$ of stroke cases. Nevertheless, it is the most alarming type due to its large fatality rate and poor functional outcome (Qureshi et al., 2009; Manaenko et al., 2013). ICH results from rupture of blood vessels in the brain. The quick release of blood into the parenchyma leads to substantial mechanical damage that may only be somewhat ameliorated by limiting hematoma volume (Mayer et al., 2008). Meanwhile, secondary damage ensues due to toxic effects of released blood components, such as thrombin, as well as erythrocyte rupture (for example, iron-catalyzed free radical reactions). These events, including disruption of the blood-brain barrier (BBB) as well as growth of edema and inflammation, are currently therapeutic targets for ICH (Fingas et al., 2009; Keep et al., 2012). 
Therapies such as angiogenesis have been suggested for $\mathrm{ICH}$. Growing evidence suggests that after $\mathrm{ICH}$, angiogenesis is upregulated in damaged brain tissue of the peri-hematoma area, leading to compensatory cerebral vascular network remodeling (Teng et al., 2008; Lei et al., 2015). Past reports showed that many angiogenic aspects could ease ischemic brain injury, elevate focal blood flow and enhance neurological results, which indicate that recently formed microvessels are indeed functional (Hao et al., 2011; Shen et al., 2013). Blood vessels are a significant scaffolding factor that assist with the migration of neurons to damaged brain areas and supply trophic material to new neurons (Kojima et al., 2010; Lei et al., 2013). The molecules that encourage neurogenesis and angiogenesis after brain injuries (e.g., ICH) are still unidentified.

Recently, a highly conserved family of adiponectin paralogs, C1q/tumor necrosis factor(TNF)-related proteins (CTRPs), was identified. Each of the 15 known members (CTRP1-CTRP15) is made up of four separate domains including an N-terminal signal peptide, a short variable domain, a collagen-like domain, and a C-terminal C1q-like globular domain (Ahima et al., 2006). Both CTRPs and adiponectin are a part of the C1q/TNF protein superfamily, which proceeds to increase in size as more C1q domain proteins are identified (Yi et al., 2012). The CTRP family members exhibit broadly diverse physiological functions, including regulation of metabolism, protection against endothelial dysfunction and angiogenesis.

CTRP3 is ubiquitously expressed in adipocytes, cartilagocytes, monocytes, fibroblasts, placenta, osteosarcoma, chondroblastoma, giant cell tumor, colon, small intestine, pancreas, kidney, thymus, ovary and in brain (Schaffler and Buechler, 2012). Most importantly, CTRP3 is the only one whose biological functions have been identified (Peterson et al., 2010). It was found that CTRP3 can encourage in vitro endothelial cell proliferation and migration (Akiyama et al., 2007). But, the role of CTRP3 in promoting angiogenesis in ICH-induced brain injury is not yet known. Further, whether or not CTRP3, an important member of the most recently discovered adipokine family, works as a mediator or inhibitor of ICH has not been studied previously. Therefore, the goals of this research were: (1) to investigate the effects of exogenous CTRP3 in an ICH rat model; (2) to determine whether CTRP3 administration promotes angiogenesis after $\mathrm{ICH}$; and (3) to elucidate the role of CTRP3 in pathogenesis of ICH.

\section{MATERIALS AND METHODS}

\section{Experimental Animals}

All animal studies were given approval by the Chongqing Medical University Biomedical Ethics Committee. All experimental procedures were done in accordance with the National Institutes of Health Guide for the Care and Use of Laboratory Animals. All efforts were made to minimize the number of animals used and their suffering. A total of 115 adult male Sprague-Dawley rats (60-80 d old, 240-300 g) were used for the in vivo study.

\section{Establishment of Intracerebral Hemorrhage Model}

ICH was induced by an intrastriatal blood infusion method as described previously (Ni et al., 2015). Briefly, rats were deeply anesthetized with chloral hydrate $(350 \mathrm{mg} / \mathrm{kg}$, intraperitoneal injection) and placed in a stereotaxic frame (Kopf Instruments, Tujunga, CA, USA). After removing the hair and cleaning the scalp, the skin was incised. A burr hole was drilled $0.2 \mathrm{~mm}$ anterior and $3.0 \mathrm{~mm}$ lateral right of bregma. Whole blood $(50 \mu \mathrm{L})$, which was drawn from the femoral artery, was infused manually over $10 \mathrm{~min}$ via a $26 \mathrm{G}$ needle inserted into the striatum at a depth of $5.8 \mathrm{~mm}$ below the surface of the skull. After $10 \mathrm{~min}$, the needle was steadily taken out for $5 \mathrm{~min}$ followed by the sealing of the burr hole with a sterilized medical bone wax. The wound was cleaned, and the scalp was sutured. The animals were given time to heal in their cages. During the recovery period, the animals had unlimited access to nourishment.

\section{In vivo Experiments}

Rats were given free access to food and water in an optimal environment preceding the operation. Three in vivo experiments were performed as described below.

\section{Experiment 1}

Adult rats were split at random into the following four groups: sham-operated (sham) group, ICH group, ICH + vehicle group and $\mathrm{ICH}+$ recombinant CTRP3 (rCTRP3, Chimerigen, USA) group. rCTRP3 was injected intracerebroventricularly (80 $\mu \mathrm{g} / \mathrm{kg}$ ) $30 \mathrm{~min}$ after $\mathrm{ICH}$ and then three times per week until the animals were killed. Neurological deficits (assessed by a modified Garcia test, beam walking test and wire hanging test), hematoma volume, BBB integrity and brain edema were measured 7 days after ICH, and samples for western blot, qRT-PCR and immunohistochemistry were collected.

\section{Experiment 2}

Adult rats were split at random into the following four groups: sham-operated (sham) group, ICH group, ICH + null vector control (Lenti.null) group, ICH + lentivirus overexpression of CTRP3 (Lenti.CTRP3) group. Fourteen days after Lenti.CTRP3 intracerebroventricular injection, the rats underwent $\mathrm{ICH}$. Neurological deficits, hematoma volume, BBB integrity and brain edema were measured 7 days after ICH, and samples for western blot, qRT-PCR and immunohistochemistry were collected.

\section{Experiment 3}

Adult rats were split at random into the following four groups: ICH group, ICH + rCTRP3 group, ICH + rCTRP3 + compound $\mathrm{C}$ (Com.C) group (AMP-activated protein kinase (AMPK) axis inhibitor, $20 \mu \mathrm{g} / \mathrm{kg}$, intracerebroventricular injection, 3 times per week), and $\mathrm{ICH}+\mathrm{rCTRP} 3+\mathrm{CBO}-$ P11 (CBO) group (vascular endothelial growth factor (VEGF) inhibitor $40 \mu \mathrm{g} / \mathrm{kg}$, intracerebroventricular injection, 3 times per week). Neurological deficits and BBB integrity were 
measured 7 days after $\mathrm{ICH}$, and samples for western blot and immunohistochemistry were collected.

\section{Lentivirus-CTRP3 Gene Transfer in the Rat Brain}

Adult rats were anesthetized with chloral hydrate $(350 \mathrm{mg} / \mathrm{kg}$ intraperitoneal injection) and then placed in a Kopf stereotactic frame. A burr hole was bored in the pericranium $0.9 \mathrm{~mm}$ lateral to the sagittal suture and $1.9 \mathrm{~mm}$ posterior to the coronal suture. A $10 \mu \mathrm{L}$ microinjection pump (WPI Inc., Sarasota, FL, USA) was stereotactically inserted $3.5 \mathrm{~mm}$ deeper into the cortex. A $5 \mu \mathrm{L}$ viral suspension consisting of $1 \times 10^{9}$ genomic copies of the lentivirus-CTRP3 (Lenti-CTRP3) gene, which was injected ipsilaterally into the right lateral cerebral ventricle at a rate of $0.2 \mu \mathrm{L} / \mathrm{min}$. The needle was taken out after $15 \mathrm{~min}$ of injection. The animals then were allowed to heal and brought back to their cages.

\section{Behavioral Assessment}

Behavioral functions were measured using a modified Garcia test, beam walking test, and wire hanging test 7 days after ICH in a blind fashion (Chen et al., 2013). In the modified Garcia test, rats were given a score of $0-18$. The scoring system consisted of six tests, with possible scores of $0-3$ $(0=$ worst; 3 = best $)$. The minimum score was 0 and the maximum was 18 . Beam walking and wire hanging tests utilized bridges (550 $\mathrm{cm}$ wire or $590 \mathrm{~cm}$ beam) between two platforms on which the rats were placed in the center. Rats were assessed according to six criteria that described if the animal could reach the platform and use its limbs in a symmetrical manner and were then given a score of $0-5$ (normal). The average of three trials per test for each animal was calculated.

\section{BBB Permeability}

Seven days after ICH, rats were intravenously injected with $2 \%$ Evans blue dye $(4 \mathrm{~mL} / \mathrm{kg}$; Sigma-Aldrich, St. Louis, MO, USA). Three hours later, the amount of extravasated Evans blue dye in the hemorrhagic brain hemispheres was evaluated by spectrophotometry (Thermo Scientific, MA, USA) at $620 \mathrm{~nm}$ (Cai et al., 2015).

\section{Brain Water Content}

Seven days after ICH, the cerebral hemisphere was cut into 4-mm thick blocks around the needle track. Brain tissues were immediately weighed using an analytical balance and heated at $100^{\circ} \mathrm{C}$ for $24 \mathrm{~h}$ to obtain the dry weight. The water content was calculated using the following formula: (wet weight- dry weight)/wet weight $\times 100 \%$.

\section{Hematoma Volume}

Hematoma volume was evaluated using a spectrophotometric hemoglobin assay 7 days after the ICH operation (Ma et al., 2014).

\section{Western Blot Analysis}

Total protein was extracted from the peri-hematoma area of the rat striatum using cell lysis buffer supplemented with proteinase and phosphatase inhibitors. Cell lysates were split by 10\% SDSPAGE and transferred to polyvinylidene fluoride membranes. Then, the membranes were blocked in 5\% non-fat milk TBST buffer for $1.5 \mathrm{~h}$ at room temperature. The membranes were incubated in primary antibody overnight at $4^{\circ} \mathrm{C}$ and in secondary antibody for $1 \mathrm{~h}$ at room temperature. Dilutions for primary antibodies were the following: anti-vascular endothelial growth factor (VEGFA; 1:1000, Abcam, Cambridge, MA, USA), antihypoxia inducing factor- $1 \alpha$ (HIF- $1 \alpha ; 1: 500$, Abcam, Cambridge, MA, USA), and anti-AMPK (phospho-thr172; 1:500, Bioworld, Dublin, OH, USA). The secondary antibody was diluted 1:5000 (Sangon Biotech, Shanghai, Co., Ltd.). The density of bands was detected using an imaging densitometer (BioRad, Foster City, CA, USA), and the gray value of bands was quantified using Quantity One 1-D analysis software (Bio-Rad).

\section{qRT-PCR}

Total RNA was removed with RNAiso Plus (TaKaRa Biotechnology, Dalian, China) using the manufacturer's instructions. Reverse transcription was done with a cDNA
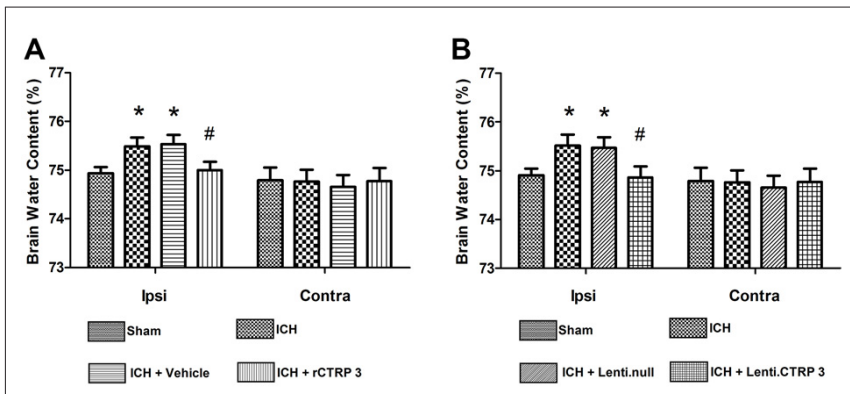

FIGURE 1 | Effects of CTRP3 treatment on brain water content 7 days after ICH. (A) Brain water content in ipsilateral (Ipsi) and contralateral (Contra) hemispheres 7 days after $\mathrm{ICH}$ in rats treated with rCTRP3. (B) Brain water content in ipsilateral (Ipsi) and contralateral (Contra) hemispheres 7 days after $\mathrm{ICH}$ in rats treated with Lenti-CTRP3. Values are mean \pm SEM. $n=8$ per group. ${ }^{*} p<0.05$ vs. sham; ${ }^{*} p<0.05$ vs. vehicle or Lenti.null. CTRP3, C1q/tumor necrosis factor-related protein-3; rCTRP3, recombinant CTRP3; Lenti.CTRP3, Lentivirus overexpression of CTRP3; ICH, intracerebral hemorrhage.

TABLE 1 | Primers used in qRT-PCR.

\begin{tabular}{|c|c|c|c|}
\hline Gene product & Forward primer & Reverse primer & Fragment size (bp) \\
\hline CTRP3 & 5'-ATGGAGGTGAGCAGAAGAGC-3' & 5'CACAGTCCCCGTITAGCAT-3' & 126 \\
\hline $\mathrm{HIF}-1 \alpha$ & 5'-CTCCCTIПTCAAGCAGCAG-3' & 5'GCTCСАТTССАТССТGTTCA-3' & 125 \\
\hline VEGFA & 5'-CGTCCTGTGTGCCCCTAAT-3' & 5'TGGCTITGGTGAGGTाTGAT-3' & 121 \\
\hline$\beta$-actin & 5'-TGTTGAGACCTTCAACACC-3' & 5'-CGCTCATTGCCGATAGTGAT-3' & 207 \\
\hline
\end{tabular}


synthesis kit (TaKaRa Biotechnology). Real-time PCR reactions were performed with TaKaRa SYBR Premix Ex Taq II (TliRnaseH Plus, TaKaRa Biotechnology) on a PCR amplifier (CFX-96 Content Real-time System). Primers (Sangon Biotech) are recorded in Table $\mathbf{1 .}$

\section{Immunohistochemistry}

Rats were killed 7 days after ICH induction by intraperitoneal injection of chloral hydrate. Immunohistochemistry was performed as previously described (Lei et al., 2013; Lapi et al., 2015). The primary antibody used was rabbit anti-rat CD31 (1:100, Abcam, USA). Total vessel densities were computed by counting 4 areas in 3 sections through the stroke region for each animal. Sections were stained with antibody against new vessel marker CD31; positive staining appeared brown. Standard quantitation was done as percent CD31-positive in the region bordering the hematoma.

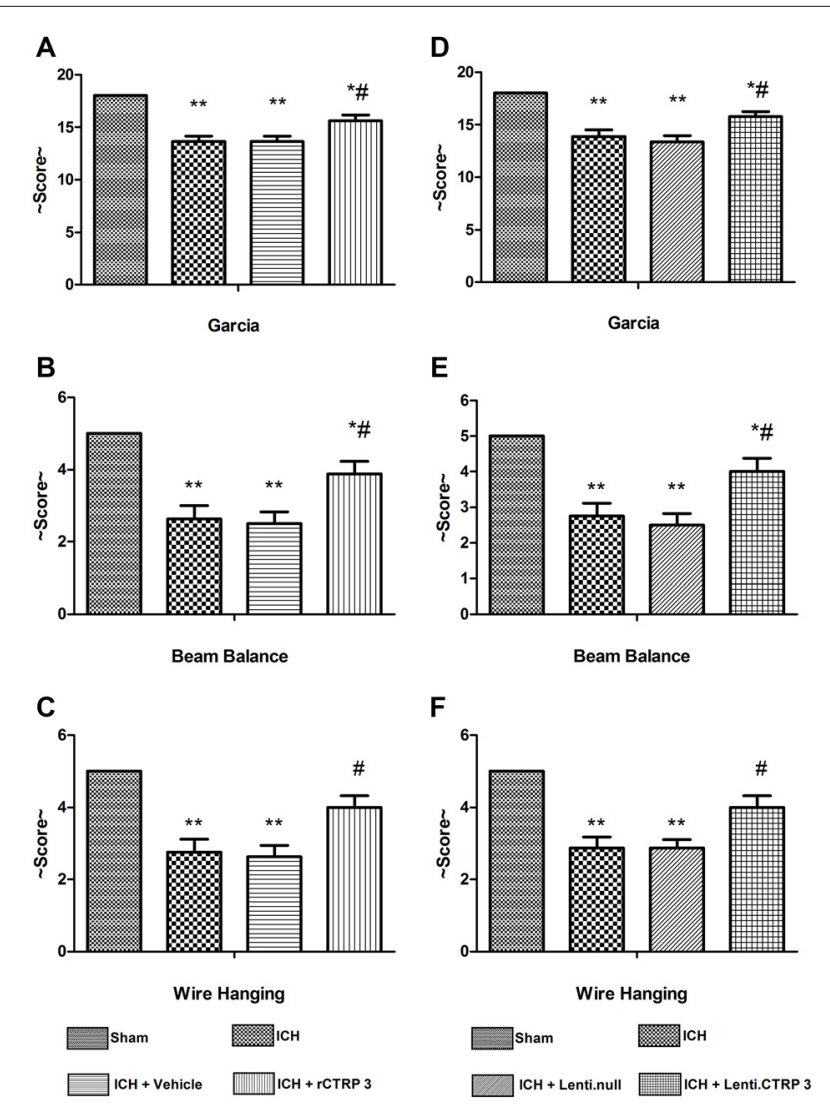

FIGURE 2 | Effects of CTRP3 treatment on neurological functions 7 days after $\mathbf{I C H}$. All animals after $\mathrm{ICH}$ showed significant neurological deficits based on performance on the modified Garcia $(\mathbf{A})$, beam balance $\mathbf{( B )}$ and wire hanging $(\mathbf{C})$ tests. Rats treated with rCTRP3 showed reduced neurological deficits in all three tests. Treatment with Lenti-CTRP3 starting 2 weeks before induction of $\mathrm{ICH}$ showed a tendency to ameliorate neurological deficits (D-F). Values are mean \pm SEM. $n=9$ per group. ${ }^{*} p<0.05$ vs. sham; ${ }^{* *} p<0.01$ vs. sham; ${ }^{\#} p<0.05$ vs. vehicle or Lenti.null. CTRP3, C1q/tumor necrosis factor-related protein-3; rCTRP3, recombinant CTRP3; Lenti-CTRP3, Lentivirus overexpression of CTRP3; $\mathrm{ICH}$, intracerebral hemorrhage.

\section{Statistical Analysis}

All data are given as mean \pm S.E.M. One-way analysis of variance (ANOVA) followed by Student's $t$ test was utilized to collate outcomes among all groups. The SPSS 17.0 software package was utilized to do all statistics. $P<0.05$ was considered statistically significant.

\section{RESULTS}

\section{CTRP3 Reduced Brain Edema and Improved Functional Outcomes after ICH}

Quantification of brain water content showed that ICH rats had significantly greater edema in the ipsilateral hemisphere than sham-operated rats (Figure 1A). Brain edema in the ipsilateral hemisphere was significantly less in rCTRP3treated rats than in vehicle-treated rats (Figure 1A). In the contralateral hemisphere, rCTRP3 failed to affect brain water content (Figure 1A). Subsequently, we tested functional outcomes using a battery of behavioral tests in rats treated with $\mathrm{rCTRP} 3$ or vehicle. Neurological deficits were significantly more severe in all ICH vs. sham animals 7 days after ICH as tested by the modified Garcia test (Figure 2A), wire hanging test (Figure 2B), and beam balance test (Figure 2C). A statistically significant advancement was seen in all three neurobehavioral tests after rCTRP3 treatment.

Similarly, Lenti.CTRP3 treatment decreased brain water content in ICH rats (Figure 1B). Statistically significant neurological shortfalls were observed in all ICH vs. sham animals 7 days after ICH. Treatment with Lenti-CTRP3 tended to ameliorate neurological deficits 7 days after ICH (Figures 2D-F). Both the rCTRP3 and Lenti.CTRP3 treatments were effective.
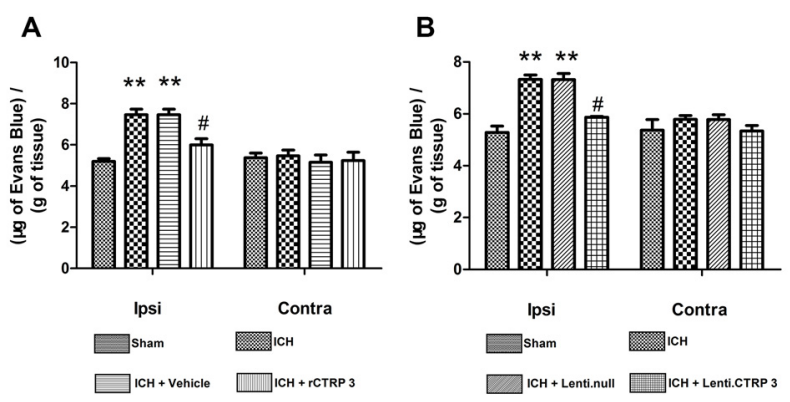

FIGURE 3 | Effects of CTRP3 treatment on ICH-induced blood-brain barrier (BBB) damage 7 days after ICH. (A) Quantification of Evans blue dye extravasation (blue staining) in the ipsilateral (Ipsi) and contralateral (Contra) hemispheres 7 days after $\mathrm{ICH}$ in rats treated with rCTRP3.

(B) Quantification of Evans blue dye extravasation (blue staining) in ipsilateral (Ipsi) and contralateral (Contra) hemispheres 7 days after $\mathrm{ICH}$ in rats treated with Lenti-CTRP3. Values are mean \pm SEM. $n=6$ per group. ${ }^{* *} p<0.01$ vs. sham; ${ }^{\#} p<0.05$ vs. vehicle or Lenti.null. CTRP3, C1q/tumor necrosis factor-related protein-3; rCTRP3, recombinant CTRP3; Lenti-CTRP3, Lentivirus overexpression of CTRP3; ICH, intracerebral hemorrhage. 
A

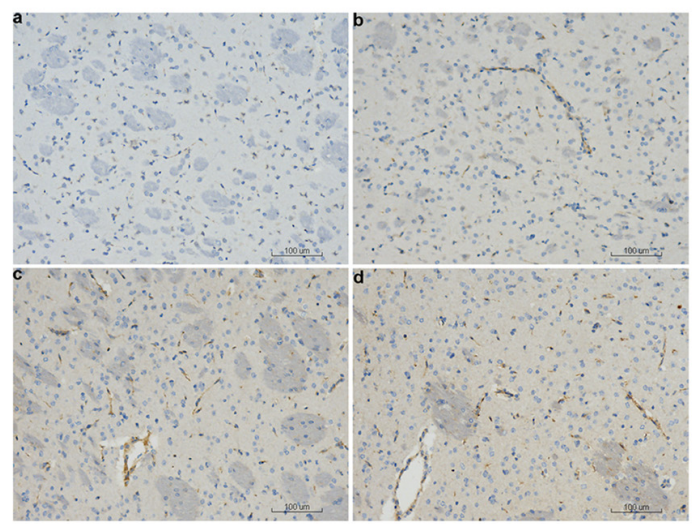

B

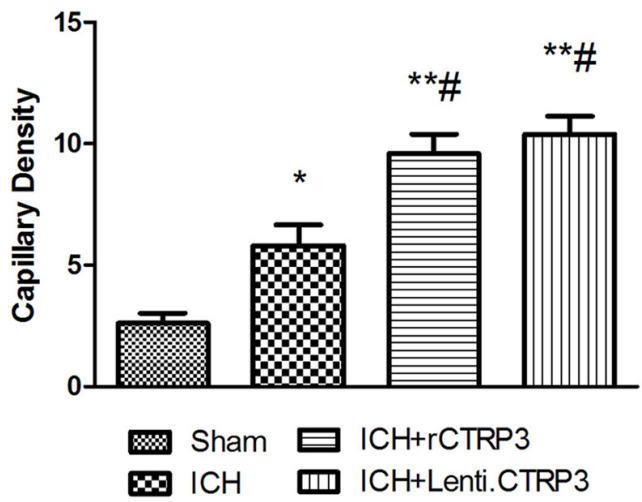

FIGURE 4 | CTRP3 encourages angiogenesis in the hematoma border zone 7 days after ICH. (A) Capillary density computed by immunohistochemical staining for CD31 (brown) in the perifocal region in ICH (b), rCTRP3 (c) and Lenti-CTRP3 (d) 7 days after ICH. A control brain section demonstrates a normal distribution of microvessels in the same region (a). Bar $=100 \mu \mathrm{m}$. (B) Bar graph showing the number of brown stained capillaries 7 days after ICH. Values are mean \pm SEM. $n=5$ per group. ${ }^{*} p<0.05$ vs. sham; ${ }^{* *} p<0.01$ vs. sham; ${ }^{*} p<0.05$ vs. ICH. CTRP3, C1q/tumor necrosis factor-related protein-3; rCTRP3, recombinant CTRP3; Lenti-CTRP3, Lentivirus overexpression of CTRP3; ICH, intracerebral hemorrhage; CD31, platelet endothelial cell adhesion molecule-1.

A

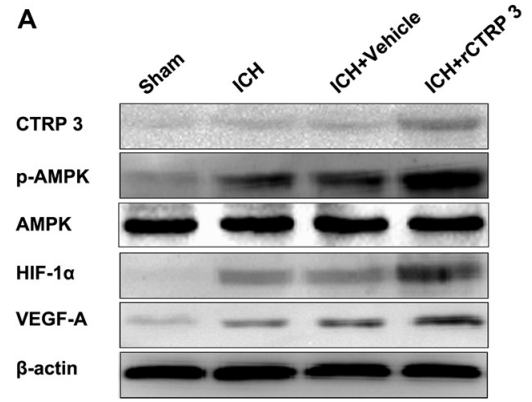

B

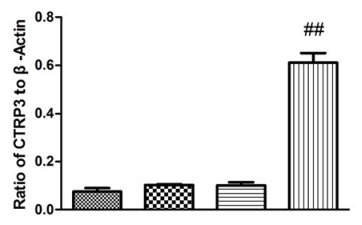

D

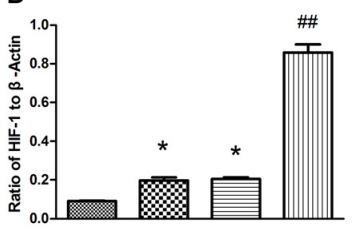

Wham

ICH + Vehicle
C

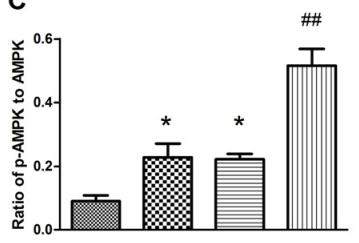

E

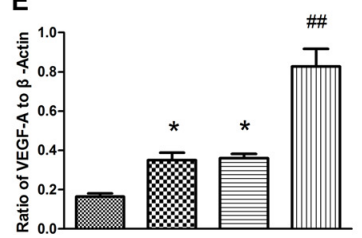

(1) ICH

$\mathrm{ICH}+\mathrm{rCTRP} 3$

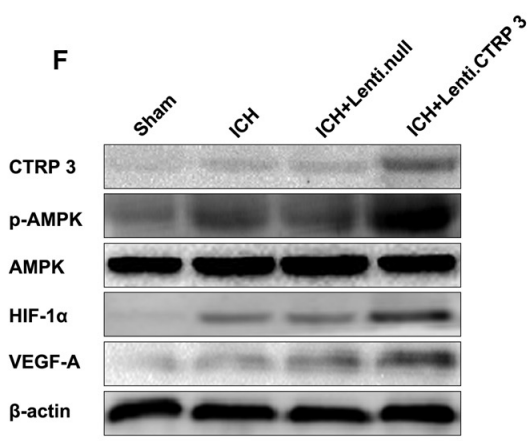

G

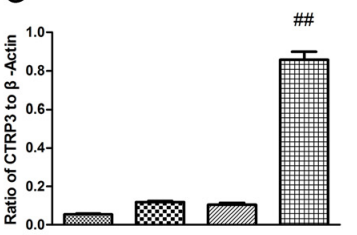

H

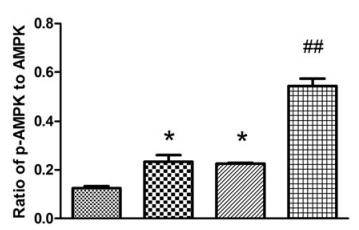

I

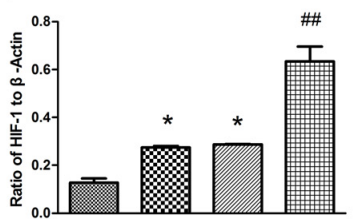

J

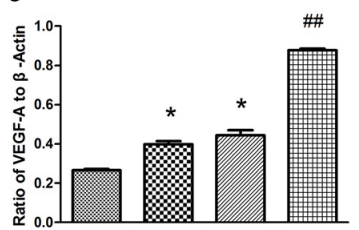

Wham

(1) $1 \mathrm{CH}$

ICH + Lenti.null $I$ ICH + Lenti.CTRP 3

FIGURE 5 | CTRP3 upregulates PAMPK/HIF-1 $\alpha /$ vascular endothelial growth factor (VEGF) axis protein expression in the hematoma border zone 7 days after ICH. (A) Western blot analysis of the effect of rCTRP3 on CTRP3, phosphorylated (p) AMP-activated protein kinase (AMPK; Thr172)/AMPK, HIF-1 $\alpha$ and VEGF-A. (B-E) Representative ratios of CTRP3, p-AMPK, HIF-1 $\alpha$ and VEGF-A protein expression. (F) Western blot analysis of the effect of Lenti-CTRP3 on CTRP3, phosphorylated (p) AMPK (Thr172)/AMPK, HIF-1 $\alpha$ and VEGF-A. (G-J) Representative ratios of CTRP3, p-AMPK, HIF-1 $\alpha$ and VEGF-A protein expression. Values are mean \pm SEM. $n=4$ per group. ${ }^{*} p<0.05$ vs. sham; \#\# $p<0.01$ vs. vehicle or Lenti.null. CTRP3, C1q/tumor necrosis factor-related protein-3; rCTRP3, recombinant CTRP3; Lenti-CTRP3, Lentivirus overexpression of CTRP3; ICH, intracerebral hemorrhage. 


\section{CTRP3 Reduced Disruption of the BBB}

We measured $\mathrm{BBB}$ permeability by Evans blue extravasation in $\mathrm{ICH}$ rats. Significant accumulation of Evans Blue stain was seen in the ipsilateral hemisphere of $\mathrm{ICH}$ in comparison to sham animals (Figure 3A). Treatment with rCTRP3 significantly reduced the quantity of stain in the ipsilateral hemisphere in comparison to the vehicle 7 days after ICH (Figure 3A). Rats were similarly protected when treatment with LentiCTRP3 also caused a significant reduction in BBB permeability (Figure 3B).

\section{Effect of CTRP3 on the Hematoma Volume-Hemoglobin Assay}

Neither recombinant nor lentivirus CTRP3 treatment had any obvious effect on hemorrhage volume at 7 days (data not shown).

\section{CTRP3 Promotes Angiogenesis and Activates the AMPK/HIF-1 $\alpha /$ VEGF Axis in the ICH Brain}

Angiogenesis is a vital part in beginning brain recovery after ICH. Having shown CTRP3 as an innovative adipokine involved in recovery following $\mathrm{ICH}$, we assessed angiogenic effects of CTRP3. As illustrated in Figures 4A,B, CTRP3 treatment significantly enhanced the amount of CD31positive capillary vessels in the zone bordering the hematoma 7 days after $\mathrm{ICH}$, pointing to the observation that CTRP3 encourages vessel formation following $\mathrm{ICH}$.

To further figure out the signaling pathways in charge of increased angiogenesis after CTRP3 treatment, many critical mediators and cytokines required for angiogenesis were tested. rCTRP3 significantly enhanced AMPK phosphorylation and increased HIF-1 $\alpha$ and VEGF expression (Figures 5A-E). Similar changes in mediator and cytokine expression were found when animals were treated with lentivirus CTRP3 (Figures 5F-J). The results from qRT-PCR were in agreement with those from Western blots (Figure 6). These outcomes imply that CTRP3 promotes angiogenesis after ICH possibly through the AMPK/HIF-1 $\alpha /$ VEGF axis.

\section{Inhibiting AMKP or VEGF Activation Attenuated Effects of Recombinant CTRP3}

To determine whether the AMPK/HIF-1 $\alpha /$ VEGF signaling pathway is accountable for CTRP3 protection seen in vivo, Com.C (AMPK axis inhibitor) or CBO-P11 (VEGF inhibitor) was given together with $\mathrm{rCTRP} 3$ via the right lateral cerebral ventricle, and neurological function, BBB integrity and capillary density were assessed 7 days after ICH. As shown in Figures 7A-D, blocking AMPK activation abolished rCTRP3-induced pAMPK, HIF-1 $\alpha$ and VEGF upregulation, and blocking VEGF activation only abolished rCTRP3-induced VEGF upregulation. In addition, CTRP3-induced capillary formation was entirely terminated when either AMPK or VEGF was inhibited (Figures $\mathbf{7 G}, \mathbf{H}$ ). But, the neurological
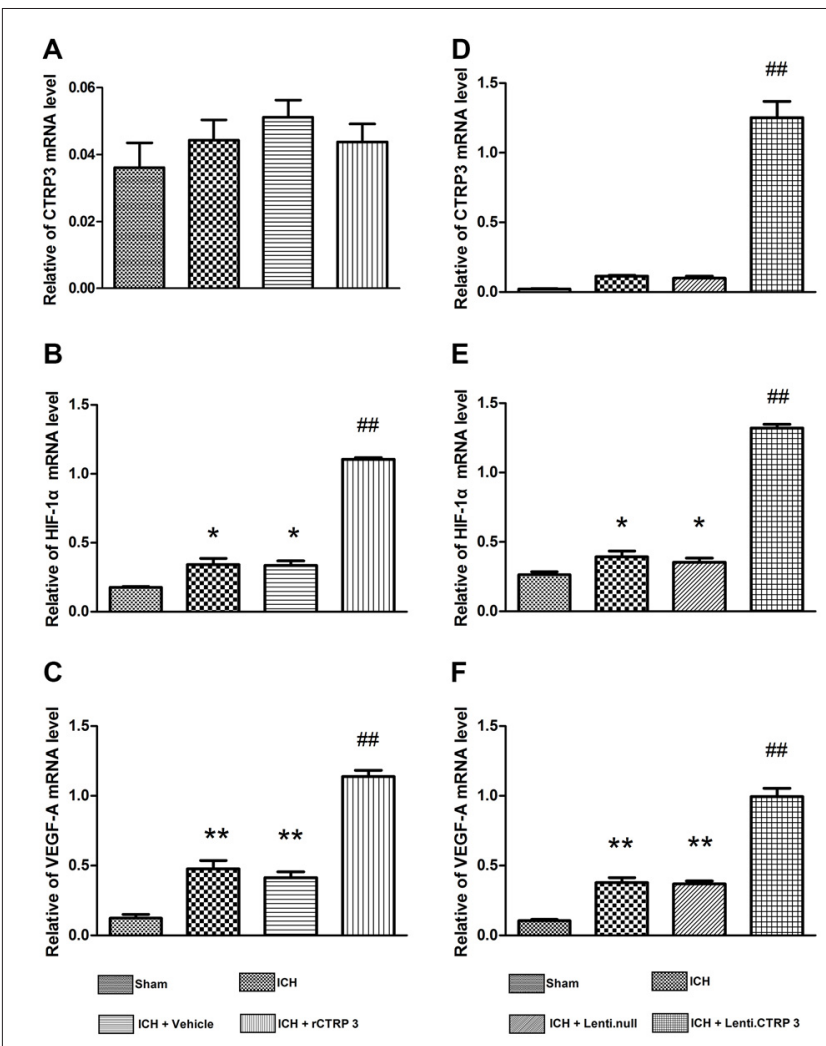

E
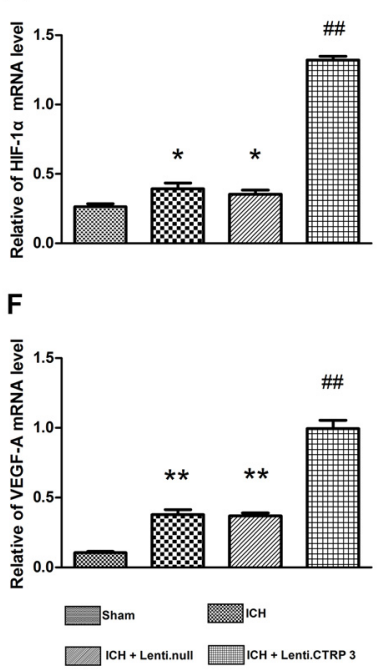

FIGURE 6 | Quantitative RT-PCR analysis of HIF-1 $\alpha$ and VEGF-A mRNA levels in the hematoma border zone 7 days after ICH. (A-C) Quantitative RT-PCR analysis of the effect of rCTRP3 on CTRP3, HIF- $1 \alpha$ and VEGF-A mRNA levels. (D-F) Quantitative RT-PCR analysis of the effect of rCTRP3 on CTRP3, HIF-1 $\alpha$ and VEGF-A mRNA levels. Values are mean \pm SEM. $n=4-6$ per group. ${ }^{*} p<0.05$ vs. sham; ${ }^{* *} p<0.01$ vs. sham; ${ }^{\# \#} p<0.01$ vs. vehicle or Lenti.null. CTRP3, C1q/tumor necrosis factor-related protein-3; rCTRP3, recombinant CTRP3; Lenti.CTRP3, Lentivirus overexpression of CTRP3; $\mathrm{ICH}$, intracerebral hemorrhage.

protective aspect of CTRP3 was completely blocked by Com.C and partially blocked by CBO-P11 (Figures 7E,F). These results imply that, though the neurological protective effect of CTRP3 is controlled significantly by AMPK/HIF$1 \alpha /$ VEGF signaling-induced angiogenesis, other mechanisms also provide neurological protection to CTRP3 against $\mathrm{ICH}$.

\section{DISCUSSION}

In the present study, we investigated the ability of CTRP3 to ameliorate secondary brain injury after $\mathrm{ICH}$ in rats. Based on our knowledge, this is the first report that investigated the effects of CTRP3 therapy in angiogenesis and brain injury after ICH. The main discoveries of the study are as follows: (1) exogenous rCTRP3 or Lenti-CTRP3 in ICH rats demonstrates the same tendency to attenuate $\mathrm{ICH}$-induced brain injury; (2) CTRP3 promotes focal angiogenesis and attenuates $\mathrm{ICH}$-induced brain edema and breakdown of the BBB; and (3) CTRP3 may exert its angiogenic effect through AMPK/HIF$1 \alpha /$ VEGF signaling. These findings imply that CTRP3 is a novel 
A

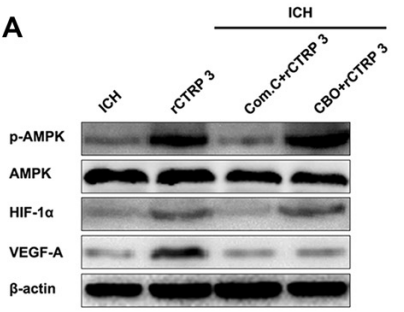

D

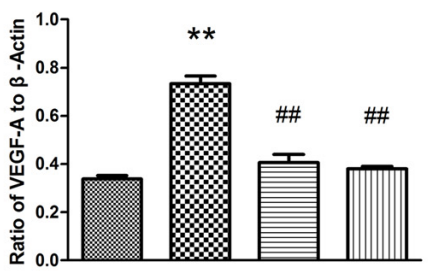

B

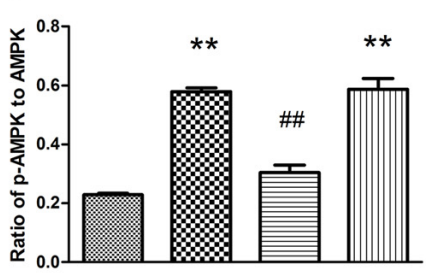

C

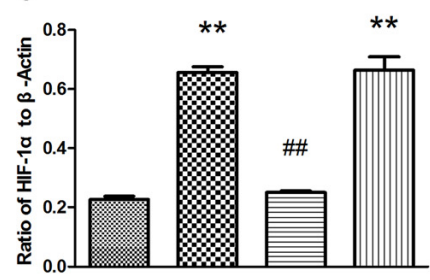

E

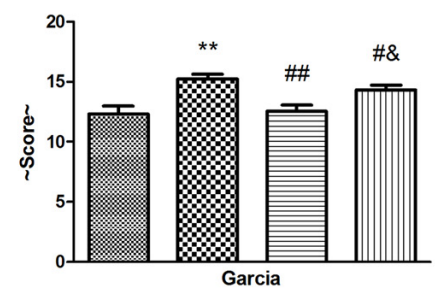

F

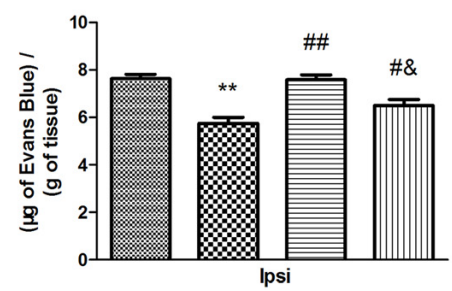

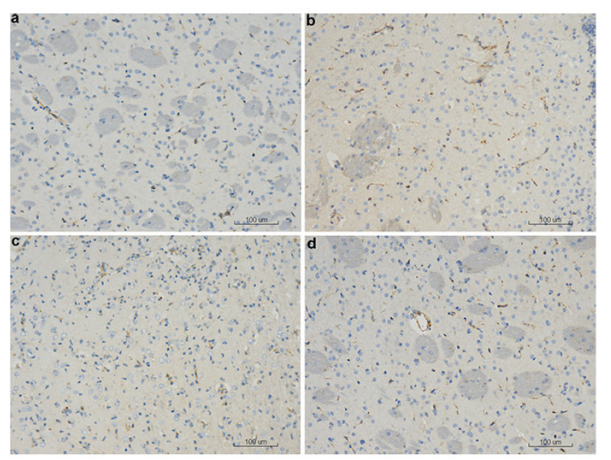

H

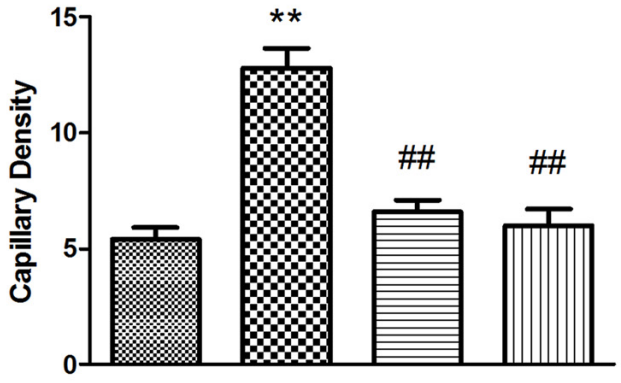

ICH

ICH + Com.C + rCTRP 3

$\mathrm{ICH}+\mathrm{CBO}+\mathrm{rCTRP} 3$

FIGURE 7 | CTRP3 promotes angiogenesis via an AMPK-dependent signaling mechanism. (A) Western blot analysis of the effects of COM.C and CBO on rCTRP3-induced p-AMPK, HIF-1 $\alpha$ and VEGF-A expression. (B-D) Representative ratios of p-AMPK, HIF-1 $\alpha$ and VEGF-A protein expression. (E) Modified Garcia test analysis of the effects of COM.C and CBO on neurological function improvement induced by rCTRP3. (F) Evans blue dye extravasation analysis of the effects of COM.C and CBO on ICH-induced BBB damage reduced by rCTRP3. (G) Immunohistochemical staining analysis of the effects of COM.C and CBO on angiogenesis induced by rCTRP3. Capillary density measured by immunohistochemical staining for CD31 (brown) in the perifocal region in ICH (a), rCTRP3 (b), COM.C (c) and $\mathrm{CBO}$ (d) 7 days after $\mathrm{ICH}$. (H) Bar graph showing the number of brown stained capillaries. Values are mean \pm SEM. $n=4-6$ per group. ${ }^{* *} p<0.01$ vs. ICH; ${ }^{\#} p<0.05$ vs. rCTRP3; $\#$ $p<0.01$ vs. rCTRP3; ${ }^{\circledR} p<0.05$ vs. COM.C. CTRP3, C1q/tumor necrosis factor-related protein-3; rCTRP3, recombinant CTRP3; Lenti-CTRP3, Lentivirus overexpression of CTRP3; ICH, intracerebral hemorrhage; COM.C, compound C; CBO, CBO-P11.

angiogenic factor that might perform a key part in encouraging angiogenesis by activating the AMPK signaling pathway during $\mathrm{ICH}$.

The prognosis of ICH is affected by multiple factors (Brown et al., 1996). Neurotrophin family, anti-oxidative mediators, anti-mitochondrial impairment or anti-inflammatory drugs contribute to functional recovery and promote neuronal survival in the central nervous system (Ip et al., 1993; Chung et al., 2013; Xu et al., 2013; Chen et al., 2015; Wei et al., 2015). Angiogenesis induced in the ischemic penumbra (Risau, 1997) as well as rapid recovery of reperfusion and oxygen supply in injured brain tissues are critical prognostic factors (Mayer et al., 1998). In this study, we provide new evidence that CTRP3 has strong angiogenic and neuroprotective aspects, implying that CTRP3 could be an innovative therapeutic target of ICH.

It is known that compensatory angiogenesis can happen in the peri-hematoma region after ICH. Angiogenesis is a stepwise procedure that includes an increase in vascular permeability, degradation of the surrounding matrix, proliferation and migration of endothelial cells, and stabilization of freshly created microvessels (Conway et al., 2001). Concerted actions of many angiogenic molecules are necessary in this procedure, and VEGF is the most vital factor during each step of angiogenesis 
(Rosenstein et al., 1998; Yancopoulos et al., 2000). A number of animal experimental studies have shown that VEGF and fibroblast growth factor treatment encourages angiogenesis with ideal efficacy and increases capillary numbers (Lavu et al., 2011; Ye, 2016). However, endogenous angiogenesis following a stroke is insufficient to reverse brain injury. Our study shows that CTRP3 successfully encourages angiogenesis and upregulates VEGF expression in the striatum ipsilateral to the hemorrhage, which leads to increased vessel density. Because CTRP3 has been established to directly encourage endothelial cell proliferation and migration but not increase tube formation (a procedure that requires complexity surpassing proliferation and migration), angiogenic factors other than VEGF are likely involved.

These findings contribute to the increasing literature on the vital part of VEGF in brain injury. In fact, VEGF levels are increased during a plethora of pathological events in the brain, implicating its essential role in brain repair processes (Cristofaro and Emanueli, 2009). VEGF binds to two receptors, VEGF receptor-1 (VEGFR-1) and VEGF receptor-2 (VEGFR2 ), through which it encourages revascularization and the mending of the $\mathrm{BBB}$ and re-establishes metabolic and trophic assistance to injured tissue (Krum et al., 2008; Shimotake et al., 2010). Future work in this field should aim to illuminate whether CTRP3 interacts with one or both receptors during ICH.

Because ICH-induced stress can change the composition, structure and distribution of the extracellular matrix, which has a vital role in creating normal brain tissue structures and is closely linked with brain injury-induced brain edema formation (Keep et al., 2012; Chung et al., 2013; Turner and Sharp, 2016), we investigated the effect of CTRP3 on BBB integrity by measuring brain water content and extravasation of Evans blue dye. We showed that angiogenesis via CTRP3 treatment resulted in preservation of the BBB. This observation in $\mathrm{ICH}$ extends past discoveries from other models of brain injury. In a model of cerebral ischemia, VEGF bound to VEGFR-2, which is expressed predominantly on activated astrocytes in the central nervous system, and encouraged revascularization and repair of the $\mathrm{BBB}$ by giving metabolic and trophic assistance to injured tissue (Krum et al., 2008; Shimotake et al., 2010; Hao et al., 2011). In a rat model of stroke, VEGF enhanced angiogenesis in the ischemic brain and reduced neurological deficits during recovery (Zhang et al., 2000). But, we were not able to demonstrate any effect of CTRP3 treatment on hematoma volume. We speculate that this may be due to use of a bloodinduced ICH model.

It is important to note that the CTRP3-treated rats manifested not only greatly strengthened angiogenesis and enhanced BBB preservation but also diminished neurological deficits. There are several possibilities for this improvement. First, the great angiogenic effect of CTRP3 in the border zone may help in restoring the blood flow, thereby rescuing dying neurons around the hematoma. Second, angiogenesis may assist new neurons migrating to damaged brain regions and give trophic material to these cells (Kojima et al., 2010; Lei et al., 2013). Finally, CTRP3 may promote differentiation of neural stem cells into neurons. Such intriguing possibilities all merit express study.

Around the hematoma, ischemia and hypoxia are apparent in ICH and this stress activates the AMPK and HIF1 signaling pathway, which induces the AMPK phosphorylation in human umbilical vein endothelial cells and promotes the recruitment, migration, proliferation and differentiation of endothelial cells (Nagata et al., 2003). In ischemic mice hindlimbs, AICAR, an AMPK antagonist induces expression of endogenous HIF target gene VEGF, but dominant-negative AMPK abolishes this expression at both steady state mRNA and protein levels (Ouchi et al., 2005). These data suggest that AMPK signaling is likely to regulate the expression of VEGF and promote angiogenesis in response to ischemic injury.

CTRP3 is a paralog of adiponectin, and it is well received that adiponectin encourages angiogenesis through activation of AMPK signaling (Shimano et al., 2010). Yet, whether CTRP3 encourages angiogenesis in cerebral tissue through the same pathway is not yet known. More important, inhibiting AMPK phosphorylation by Com.C greatly eradicated CTRP3-induced HIF- $1 \alpha$ and VEGF expression and blocked the angiogenic effect of CTRP3. These findings defend the existence of an AMPKdependent mechanism for angiogenic effects of CTRP3.

\section{CONCLUSION}

We conclude that CTRP3, a key member of a newly identified adipokine family, upregulates expression of angiogenic cytokines and induces robust angiogenesis, which led to enhanced preservation of the $\mathrm{BBB}$ and reduction of neurological deficits after ICH. The effect is mediated by the AMPK signaling pathway. These findings suggest that CTRP3 plays a positive role during ICH and has therapeutic potential.

\section{AUTHOR CONTRIBUTIONS}

SW, YaZ, YB, LL and YoZ: conceived and designed the experiments. SW, YaZ, YB and SY: conducted the experiments. SW and YaZ: analyzed the results. SW, YC and JZ: contributed materials and analysis tools. SW and YaZ: wrote the article. SW and YaZ: contributed equally to this study. All authors reviewed the manuscript.

\section{FUNDING}

This work was supported by The National Natural Science Foundation of China (81271460 and 81671158), Natural Science Youth Foundation of China (No. 81301125), the Medical scientific research projects of Chongqing (20120221) and the Natural Science Foundation of Chongqing Science and Technology Committee, China (No. cstc2015jcyjA10048).

\section{ACKNOWLEDGMENTS}

We are grateful to Key Laboratory of Neurobiology (Chongqing, China) for technical guidance and partial sponsorship. 


\section{REFERENCES}

Ahima, R. S., Qi, Y., Singhal, N. S., Jackson, M. B., and Scherer, P. E. (2006). Brain adipocytokine action and metabolic regulation. Diabetes 55, S145-S154. doi: 10. 2337/db06-s018

Akiyama, H., Furukawa, S., Wakisaka, S., and Maeda, T. (2007). CTRP3/cartducin promotes proliferation and migration of endothelial cells. Mol. Cell. Biochem. 304, 243-248. doi: 10.1007/s11010-007-9506-6

Brown, R. D., Whisnant, J. P., Sicks, J. D., O'Fallon, W. M., and Wiebers, D. O. (1996). Stroke incidence, prevalence and survival: secular trends in Rochester, Minnesota, through 1989. Stroke 27, 373-380.

Cai, P., Luo, H., Xu, H., Zhu, X., Xu, W., Dai, Y., et al. (2015). Recombinant ADAMTS 13 attenuates brain injury after intracerebral hemorrhage. Stroke 46, 2647-2653. doi: 10.1161/STROKEAHA.115.009526

Chen, S., Ma, Q., Krafft, P. R., Chen, Y., Tang, J., Zhang, J., et al. (2013). $\mathrm{P} 2 \mathrm{X} 7$ receptor antagonism inhibits p38 mitogen-activated protein kinase activation and ameliorates neuronal apoptosis after subarachnoid hemorrhage in rats. Crit. Care Med. 41, e466-e474. doi: 10.1097/CCM.0b013e31829a 8246

Chen, D., Wei, X., Zou, J., Wang, R., Liu, X., Xu, X., et al. (2015). Contradirectional expression of serum homocysteine and uric acid as important biomarkers of multiple system atrophy severity: a cross-sectional study. Front. Cell. Neurosci. 9:247. doi: 10.3389/fncel.2015.00247

Chung, C. Y., Yang, J. T., and Kuo, Y. C. (2013). Polybutylcyanoacrylate nanoparticles for delivering hormone response element-conjugated neurotrophin-3 to the brain of intracerebral hemorrhagic rats. Biomaterials 34 , 9717-9727. doi: 10.1016/j.biomaterials.2013.08.083

Conway, E. M., Collen, D., and Carmeliet, P. (2001). Molecular mechanisms of blood vessel growth. Cardiovasc. Res. 49, 507-521. doi: 10.1016/s00086363(00)00281-9

Cristofaro, B., and Emanueli, C. (2009). Possible novel targets for therapeutic angiogenesis. Curr. Opin. Pharmacol. 9, 102-108. doi: 10.1016/j.coph.2008. 11.006

Fingas, M., Penner, M., Silasi, G., and Colbourne, F. (2009). Treatment of intracerebral hemorrhage in rats with 12 h, 3 days and 6 days of selective brain hypothermia. Exp. Neurol. 219, 156-162. doi: 10.1016/j.expneurol.2009. 05.007

Hao, Q., Su, H., Palmer, D., Sun, B., Gao, P., Yang, G. Y., et al. (2011). Bone marrow-derived cells contribute to vascular endothelial growth factorinduced angiogenesis in the adult mouse brain by supplying matrix metalloproteinase-9. Stroke 42, 453-458. doi: 10.1161/STROKEAHA.110. 596452

Ip, N. Y., McClain, J., Barrezueta, N. X., Aldrich, T. H., Pan, L., Li, Y., et al. (1993). The alpha component of the CNTF receptor is required for signaling and defines potential CNTF targets in the adult and during development. Neuron 10, 89-102. doi: 10.1016/0896-6273(93)90245-m

Keep, R. F., Hua, Y., and Xi, G. (2012). Intracerebral haemorrhage: mechanisms of injury and therapeutic targets. Lancet Neurol. 11, 720-731. doi: 10.1016/s14744422(12)70104-7

Kojima, T., Hirota, Y., Ema, M., Takahashi, S., Miyoshi, I., Okano, H., et al. (2010). Subventricular zone-derived neural progenitor cells migrate along a blood vessel scaffold toward the post-stroke striatum. Stem Cells 28, 545-554. doi: 10.1002/stem.306

Krum, J. M., Mani, N., and Rosenstein, J. M. (2008). Roles of the endogenous VEGF receptors flt-1 and flk-1 in astroglial and vascular remodeling after brain injury. Exp. Neurol. 212, 108-117. doi: 10.1016/j.expneurol.2008.03.019

Lapi, D., Vagnani, S., Sapio, D., Mastantuono, T., Boscia, F., Pignataro, G., et al. (2015). Effects of bone marrow mesenchymal stem cells (BMMSCs) on rat pial microvascular remodeling after transient middle cerebral artery occlusion. Front. Cell. Neurosci. 9:329. doi: 10.3389/fncel.2015. 00329

Lavu, M., Gundewar, S., and Lefer, D. J. (2011). Gene therapy for ischemic heart disease. J. Mol. Cell. Cardiol. 50, 742-750. doi: 10.1016/j.yjmcc.2010. 06.007

Lei, C., Lin, S., Zhang, C., Tao, W., Dong, W., Hao, Z., et al. (2013). Activation of cerebral recovery by matrix metalloproteinase- 9 after intracerebral hemorrhage. Neuroscience 230, 86-93. doi: 10.1016/j.neuroscience.2012. 11.008
Lei, C., Zhang, S., Cao, T., Tao, W., Liu, M., and Wu, B. (2015). HMGB1 may act via RAGE to promote angiogenesis in the later phase after intracerebral hemorrhage. Neuroscience 295, 39-47. doi: 10.1016/j.neuroscience.2015. 03.032

Ma, Q., Chen, S., Hu, Q., Feng, H., Zhang, J. H., and Tang, J. (2014). NLRP3 inflammasome contributes to inflammation after intracerebral hemorrhage. Ann. Neurol. 75, 209-219. doi: 10.1002/ana.24070

Manaenko, A., Lekic, T., Ma, Q., Zhang, J. H., and Tang, J. (2013). Hydrogen inhalation ameliorated mast cell-mediated brain injury after intracerebral hemorrhage in mice. Crit. Care Med. 41, 1266-1275. doi: 10.1097/CCM. 0b013e31827711c9

Mayer, S. A., Brun, N. C., Begtrup, K., Broderick, J., Davis, S., Diringer, M. N., et al. (2008). Efficacy and safety of recombinant activated factor VII for acute intracerebral hemorrhage. N. Engl. J. Med. 358, 2127-2137. doi: 10. 1056/NEJMoa0707534

Mayer, S. A., Lignelli, A., Fink, M. E., Kessler, D. B., Thomas, C. E., Swarup, R. et al. (1998). Perilesional blood flow and edema formation in acute intracerebral hemorrhage: a SPECT study. Stroke 29, 1791-1798. doi: 10.1161/01.str.29.9. 1791

Nagata, D., Mogi, M., and Walsh, K. (2003). AMP-activated protein kinase (AMPK) signaling in endothelial cells is essential for angiogenesis in response to hypoxic stress. J. Biol. Chem. 278, 31000-31006. doi: 10.1074/jbc. M300643200

Ni, W., Okauchi, M., Hatakeyama, T., Gu, Y., Keep, R. F., Xi, G., et al. (2015). Deferoxamine reduces intracerebral hemorrhage-induced white matter damage in aged rats. Exp. Neurol. 272, 128-134. doi: 10.1016/j.expneurol.2015. 02.035

Ouchi, N., Shibata, R., and Walsh, K. (2005). AMP-activated protein kinase signaling stimulates VEGF expression and angiogenesis in skeletal muscle. Circ. Res. 96, 838-846. doi: 10.1161/01.res.0000163633.10240.3b

Peterson, J. M., Wei, Z., and Wong, G. W. (2010). C1q/TNF-related protein-3 (CTRP3), a novel adipokine that regulates hepatic glucose output. J. Biol. Chem. 285, 39691-39701. doi: 10.1074/jbc.M110.180695

Qureshi, A. I., Mendelow, A. D., and Hanley, D. F. (2009). Intracerebral haemorrhage. Lancet 373, 1632-1644. doi: 10.1016/S0140-6736(09)60371-8

Risau, W. (1997). Mechanisms of angiogenesis. Nature 386, 671-674. doi: 10. 1038/386671a0

Rosenstein, J. M., Mani, N., Silverman, W. F., and Krum, J. M. (1998). Patterns of brain angiogenesis after vascular endothelial growth factor administration in vitro and in vivo. Proc. Natl. Acad. Sci. U S A 95, 7086-7091. doi: 10.1073/pnas. 95.12.7086

Schaffler, A., and Buechler, C. (2012). CTRP family: linking immunity to metabolism. Trends Endocrinol. Metab. 23, 194-204. doi: 10.1016/j.tem.2011. 12.003

Shen, L., Miao, J., Yuan, F., Zhao, Y., Tang, Y., Wang, Y., et al. (2013). Overexpression of adiponectin promotes focal angiogenesis in the mouse brain following middle cerebral artery occlusion. Gene Ther. 20, 93-101. doi: 10. 1038/gt.2012.7

Shimano, M., Ouchi, N., Shibata, R., Ohashi, K., Pimentel, D. R., Murohara, T., et al. (2010). Adiponectin deficiency exacerbates cardiac dysfunction following pressure overload through disruption of an AMPK-dependent angiogenic response. J. Mol. Cell. Cardiol. 49, 210-220. doi: 10.1016/j.yjmcc.2010. 02.021

Shimotake, J., Derugin, N., Wendland, M., Vexler, Z. S., and Ferriero, D. M. (2010) Vascular endothelial growth factor receptor-2 inhibition promotes cell death and limits endothelial cell proliferation in a neonatal rodent model of stroke. Stroke 41, 343-349. doi: 10.1161/STROKEAHA.109.564229

Teng, H., Zhang, Z. G., Wang, L., Zhang, R. L., Zhang, L., Morris, D., et al. (2008). Coupling of angiogenesis and neurogenesis in cultured endothelial cells and neural progenitor cells after stroke. J. Cereb. Blood Flow Metab. 28, 764-771. doi: $10.1038 /$ sj.jcbfm. 9600573

Turner, R. J., and Sharp, F. R. (2016). Implications of MMP9 for blood brain barrier disruption and hemorrhagic transformation following ischemic stroke. Front. Cell. Neurosci. 10:56. doi: 10.3389/fncel.2016.00056

Wei, X., Gao, H., Zou, J., Liu, X., Chen, D., Liao, J., et al. (2015). Contra-directional coupling of Nur77 and Nurr1 in neurodegeneration: a novel mechanism for memantine-induced anti-inflammation and anti-mitochondrial impairment. Mol. Neurobiol. doi: 10.1007/s12035-015-9477-7 [Epub ahead of print]. 
Xu, Y. Q., Long, L., Yan, J. Q., Wei, L., Pan, M. Q., Gao, H. M., et al. (2013). Simvastatin induces neuroprotection in 6-OHDA-lesioned PC12 via the PI3K/AKT/caspase 3 pathway and anti-inflammatory responses. CNS Neurosci. Ther. 19, 170-177. doi: 10.1111/cns.12053

Yancopoulos, G. D., Davis, S., Gale, N. W., Rudge, J. S., Wiegand, S. J., and Holash J. (2000). Vascular-specific growth factors and blood vessel formation. Nature 407, 242-248. doi: 10.1038/35025215

Ye, W. (2016). The complexity of translating anti-angiogenesis therapy from basic science to the clinic. Dev. Cell 37, 114-125. doi: 10.1016/j.devcel.2016. 03.015

Yi, W., Sun, Y., Yuan, Y., Lau, W. B., Zheng, Q., Wang, X., et al. (2012). C1q/tumor necrosis factor-related protein-3, a newly identified adipokine, is a novel antiapoptotic, proangiogenic and cardioprotective molecule in the ischemic mouse heart. Circulation 125, 3159-3169. doi: 10.1161/CIRCULATIONAHA. 112.099937
Zhang, Z. G., Zhang, L., Jiang, Q., Zhang, R., Davies, K., Powers, C., et al. (2000). VEGF enhances angiogenesis and promotes blood-brain barrier leakage in the ischemic brain. J. Clin. Invest. 106, 829-838. doi: 10.1172/jci9369

Conflict of Interest Statement: The authors declare that the research was conducted in the absence of any commercial or financial relationships that could be construed as a potential conflict of interest.

Copyright (c) 2016 Wang, Zhou, Bo, Li, Yu, Chen, Zhu and Zhao. This is an openaccess article distributed under the terms of the Creative Commons Attribution License (CC BY). The use, distribution and reproduction in other forums is permitted, provided the original author(s) or licensor are credited and that the original publication in this journal is cited, in accordance with accepted academic practice. No use, distribution or reproduction is permitted which does not comply with these terms. 